\title{
Damping rate of a fermion in ultradegenerate chiral matter
}

\author{
Stefano Carignano \\ Departament de Física Quàntica i Astrofísica and Institut de Ciències del Cosmos, \\ Universitat de Barcelona, Martí i Franquès 1, 08028 Barcelona, Catalonia, Spain \\ Cristina Manuel \\ Instituto de Ciencias del Espacio (ICE, CSIC), C. Can Magrans s.n., \\ 08193 Cerdanyola del Vallès, Catalonia, Spain \\ and Institut d'Estudis Espacials de Catalunya (IEEC), Carrer del Gran Capità 2-4, \\ Edifici Nexus, 08034 Barcelona, Spain
}

(Received 22 November 2018; revised manuscript received 30 April 2019; published 24 May 2019; corrected 22 May 2020)

\begin{abstract}
We compute the damping rate of a fermion propagating in a chiral plasma when there is an imbalance between the densities of left- and right-handed fermions, after generalizing the hard thermal loop resummation techniques for these systems. In the ultradegenerate limit, for very high energies, the damping rate of this external fermion approaches a constant value. Closer to the two Fermi surfaces, however, we find that the rate depends on both the energy and the chirality of the fermion, being higher for the predominant chirality. This comes out as a result of its scattering with the particles of the plasma, mediated by the exchange of Landau damped photons. In particular, we find that the chiral imbalance is responsible for a different propagation of the left and right circular polarized transverse modes of the photon and that a chiral fermion interacts differently with these two transverse modes. We argue that spontaneous radiation of energetic fermions is kinematically forbidden and discuss the time regime where our computation is valid.
\end{abstract}

DOI: 10.1103/PhysRevD.99.096022

\section{INTRODUCTION}

The study of chiral matter characterized by different densities of right- and left-handed massless fermions has received increasing attention in the last years. This is due to its wide range of applications, from the description of the quark-gluon plasma produced in relativistic heavy ion collisions to the so-called Weyl and Dirac semimetals in condensed matter physics, as well as its potential relevance for cosmological and astrophysical scenarios [1-7]. Systems of this type exhibit a number of unique features of extreme interest. Novel transport phenomena associated with the quantum chiral anomaly are manifested at a macroscopic level [8-11]. Further, the breaking of parity $P$ and $C P$, where $C$ stands for charge conjugation, has also a clear effect on the quasiparticles and collective modes propagating in the plasma.

In this paper, we focus our attention on the damping rate of a fermion propagating in chiral matter, whose inverse can typically be interpreted as the quasiparticle lifetime.

Published by the American Physical Society under the terms of the Creative Commons Attribution 4.0 International license. Further distribution of this work must maintain attribution to the author(s) and the published article's title, journal citation, and DOI. Funded by SCOAP ${ }^{3}$.
As it is already known for the similar case of a plasma without chiral imbalance, at finite temperature the damping rate turns out to be infrared divergent even after taking into account screening corrections of the electromagnetic interactions, and only nonperturbative approaches such as that of Ref. [12] lead to a nonexponential fermion decay law. In this work, we will instead focus on the case of a vanishing temperature but finite density. In this case, the result for the damping rate is instead finite, and for the case of a regular ultradegenerate plasma the relativistic Fermi sea was found to be stable [13-15]. The magnetic unscreened interaction nevertheless leads to a non-Fermi liquid behavior of the relativistic plasma $[14,16]$.

Let us now turn to the specific case of a plasma with a chiral imbalance. As one might expect, in this case, the damping rate for a fermion will depend on its chirality. First of all, the fermion scatters with the particles in the plasma, subject to the Pauli blocking effects, which are different depending on its chirality. But it is also important to note that the chiral imbalance affects the photon mediated interactions, by making the left- and right-handed circular polarized transverse photon modes propagate differently than in a medium without chiral imbalance. In the following, we will show this explicitly by extending the resummation methods developed for the study of QCD and QED relativistic plasmas $[17,18]$ to systems with chiral 
imbalance. Our explicit computation also shows that a chiral fermion interacts differently with the two circular polarized photons, a novel effect that, to the best of our knowledge, has never been discussed in the literature.

Furthermore, resummed propagators in chiral plasmas contain purely imaginary poles associated with the transverse photon modes, leading to an instability that results in an exponential growth of the associated gauge fields [19]. This is similar to the appearance of instabilities in a spatially anisotropic system, a topic that has been discussed at length in the context of heavy-ion collisions [20]. Accordingly, the timescale associated with the exponential growth of the fields will delimit the region in which perturbative calculations like the ones discussed in this work are applicable.

This paper is organized as follows. In Sec. II, we show how to extend the hard thermal loop (HTL) resummation scheme in plasmas with chiral imbalance. The most relevant effect comes in the splitting of the transverse modes into left- and right-handed circular polarized modes. We present the dispersion modes associated with two stable collective modes, together with their corresponding spectral functions. We also discuss the presence of unstable gauge modes. In Sec. III, we apply this extended resummation program to the computation of the damping rate of a fermion in a chiral imbalanced plasma at vanishing temperature. We finally discuss our conclusions in Sec. IV. We use natural units ( $\hbar=c=k_{B}=1$ ) throughout, and we denote by $e=\sqrt{4 \pi \alpha}$ the QED coupling constant.

\section{HTL AND COLLECTIVE MODES FOR A CHIRAL PLASMA}

Let us consider an energetic fermion crossing a chiral QED plasma, characterized by having different chemical potentials associated with left- and right-handed populations of fermions, $\mu_{L} \neq \mu_{R}$. We introduce a chiral chemical potential, defined as $\mu_{5}=\frac{1}{2}\left(\mu_{R}-\mu_{L}\right)$, to quantify this imbalance and assume it without loss of generality to be positive. We also consider the presence of a vectorial chemical potential $\mu_{V}>\mu_{5}$, so that $\mu_{R / L}=\mu_{V} \pm \mu_{5}$ are both positive.

Aside from the direct effect on the fermion propagator due to the presence of two Fermi surfaces (right and left), chiral imbalance also affects the photon propagator entering in the one-loop fermion self-energy due to the breaking of $P$ and $C P$ [21]. It is known that the leading contribution to the damping rate when the fermion energy $E$ is large comes from values of the momenta that are soft, or of the order of Debye mass [12-14], and thus resummed hard thermal or dense loop propagators have to be used [18]. In a chiral plasma, we see that the two transverse photon propagators split. In Coulomb gauge and working in the medium rest frame (we ignore gauge dependent pieces here, as they do not contribute to the imaginary part of the fermion self-energy), the photon propagator can be written as [21]

$\Delta_{\mu \nu}\left(q_{0}, \mathbf{q}\right)=\delta_{\mu 0} \delta_{\nu 0} \Delta_{L}\left(q_{0}, q\right)+\sum_{h= \pm} \mathcal{P}_{\mu \nu}^{T, h} \Delta_{T}^{h}\left(q_{0}, q\right)$,

where $q=|\mathbf{q}|$ and $h= \pm$ labels the two circular polarized transverse photon states, left and right, and we introduce

$$
\mathcal{P}_{\mu \nu}^{T, h}=\frac{1}{2}\left(\delta^{i j}-\hat{q}^{i} \hat{q}^{j}-i h \epsilon^{i j k} \hat{q}^{k}\right) \delta_{\mu i} \delta_{\nu j} .
$$

The resummed longitudinal and transverse propagators read, with the usual prescription $q_{0} \rightarrow q_{0} \pm i \eta$ for retarded and advanced quantities, respectively,

$\Delta_{L}\left(q_{0}, q\right)=\frac{1}{q^{2}+\Pi_{L}}, \quad \Delta_{T}^{h}\left(q_{0}, q\right)=\frac{1}{q_{0}^{2}-q^{2}-\Pi_{T}-h \Pi_{P}}$,

where

$$
\begin{gathered}
\Pi_{L}\left(q_{0}, q\right)=m_{D}^{2}\left(1-\frac{q_{0}}{2 q} \ln \frac{q_{0}+q}{q_{0}-q}\right), \\
\Pi_{T}\left(q_{0}, q\right)=m_{D}^{2} \frac{q_{0}^{2}}{2 q^{2}}\left[1+\frac{1}{2}\left(\frac{q}{q_{0}}-\frac{q_{0}}{q}\right) \ln \frac{q_{0}+q}{q_{0}-q}\right]
\end{gathered}
$$

are the longitudinal and transverse parts, respectively, of the photon polarization tensor $[13,14]$, and

$$
m_{D}^{2}=e^{2}\left(\frac{T^{2}}{3}+\frac{\mu_{R}^{2}+\mu_{L}^{2}}{2 \pi^{2}}\right)
$$

is the Debye mass, while

$$
\Pi_{P}\left(q_{0}, q\right)=-\frac{e^{2} \mu_{5}}{2 \pi^{2}} \frac{q_{0}^{2}-q^{2}}{q}\left[1-\frac{q_{0}}{2 q} \ln \frac{q_{0}+q}{q_{0}-q}\right]
$$

can be viewed as the anomalous hard dense loop contribution $[19,22,23]$.

The spectral functions associated with the gauge field modes are given by

$$
\begin{gathered}
\rho_{L}\left(q_{0}, q\right)=2 \operatorname{Im} \Delta_{L}\left(q_{0}+i \eta, q\right), \\
\rho_{T}^{h}\left(q_{0}, q\right)=2 \operatorname{Im} \Delta_{T}^{h}\left(q_{0}+i \eta, q\right), \quad h= \pm .
\end{gathered}
$$

The spectral functions for the resummed photon propagators for $\mu_{5}=0$ can be found in [18] (see also [14]), and we now generalize them for the case when there is chiral imbalance. As customary, they can be conveniently split into two parts, $\rho\left(q_{0}, q\right)=\rho^{>}\left(q_{0}, q\right)+\rho^{<}\left(q_{0}, q\right)$, the former being only nonvanishing above the light cone, while the latter (associated with Landau damping) is nonzero only for spacelike momenta. The longitudinal spectral function 
is the same as in a chiral symmetric plasma, while for the two transverse modes $(h= \pm)$ one finds

$\frac{\rho_{T}^{h,>}\left(q_{0}, q\right)}{2 \pi}=Z_{T}^{h}\left[\delta\left(q_{0}-\omega_{T}^{h}(q)\right)-\delta\left(q_{0}+\omega_{T}^{h}(q)\right)\right]$,

where $\omega_{T}^{h}$ are the real poles of $\Delta_{T, h}$, given by solving for $q_{0}$ the equation

$\left(q_{0}^{2}-q^{2}\right)\left[1-\frac{1}{2 q^{2}}\left(m_{D}^{2}+h C q\right)\left(1-\frac{q_{0}}{2 q} \ln \frac{q_{0}+q}{q_{0}-q}\right)\right]-\frac{m_{D}^{2}}{2}=0$

and $Z_{T}^{h}$ are the corresponding residues, given by

$Z_{T}^{h}(k)=\frac{\omega_{T, h}\left(\omega_{T, h}^{2}-k^{2}\right)}{\left(\omega_{T, h}^{2}-k^{2}\right)^{2}-m_{D}^{2} \omega_{T, h}^{2}-\frac{h C k}{2}\left(\omega_{T, h}^{2}-k^{2}\right)}$,

where

$$
C=-\frac{e^{2} \mu_{5}}{\pi^{2}}
$$

measures the chiral imbalance. The remaining part of the spectral function for values below the light cone is given by

$\frac{\rho_{T}^{h,<}}{2 \pi}=\frac{M_{h}^{2} \frac{x}{1-x^{2}} \Theta\left(1-x^{2}\right)}{\left[2 q^{2}+\frac{m_{D}^{2}}{1-x^{2}}-M_{h}^{2} Q_{1}(x)\right]^{2}+M_{h}^{4} \frac{\pi^{2} x^{2}}{4}}, \quad x=q_{0} / q$,

with $M_{h}^{2}=m_{D}^{2}+h C q$, where $Q_{1}(x)=1-\frac{x}{2} \ln \left|\frac{1+x}{1-x}\right|$.

There are both collective stable and unstable gauge modes in the chiral plasma. The real poles of $\Delta_{T, h}$ describe the existence of two different transverse stable collective modes identified by their circular polarization $h$. For small values of $q, q \ll m_{D}$, one finds the approximate behavior

$$
\omega_{T, h}^{2} \approx \omega_{p}^{2}+\frac{6}{5} k^{2}+\frac{h C}{6} k
$$

where $\omega_{p}=m_{D} / \sqrt{3}$ is the plasma frequency, while for $q \gg m_{D}$ one gets

$$
\omega_{T, h}^{2} \approx k^{2}+\frac{1}{2} m_{D}^{2}+h C \frac{m_{D}^{2}}{4 k}\left(1-\frac{1}{2} \ln \frac{8 k_{D}^{2}}{m_{D}^{2}}\right),
$$

both of which reduce to the known expressions [18] when $C \rightarrow 0$.

In Fig. 1 we show the full numerical result for the real poles of $\Delta_{T}^{h}$ for the two different polarizations. The two modes are degenerate at $q=0$ and their splitting increases as $q$ grows, up to a maximum value that is reached around $q \sim 2 m_{D}$, after which it starts decreasing again.

Aside from these real solutions, it is possible to find an additional family of poles of $\Delta_{T}^{h}$, which are purely imaginary. Indeed, for $q_{0} \ll q$ one can expand the denominator of $\Delta_{T}^{h}$ as

$$
q_{0}^{2}-q^{2}= \pm \frac{e^{2} \mu_{5}}{2 \pi^{2}} q-i \frac{\pi m_{D}^{2}}{2} \frac{q_{0}}{q},
$$

from which one gets

$$
q^{0}=-\frac{2 i}{\pi m_{D}^{2}} q^{2}\left(q-\frac{h C}{2}\right) .
$$

These purely imaginary poles give rise to the chiral plasma instability discussed in Ref. [19].

We have checked numerically that no other families of complex solutions are present. Let us also mention that the dispersion law (without the imaginary term) was employed in Ref. [24]. Our computations show that this is only valid
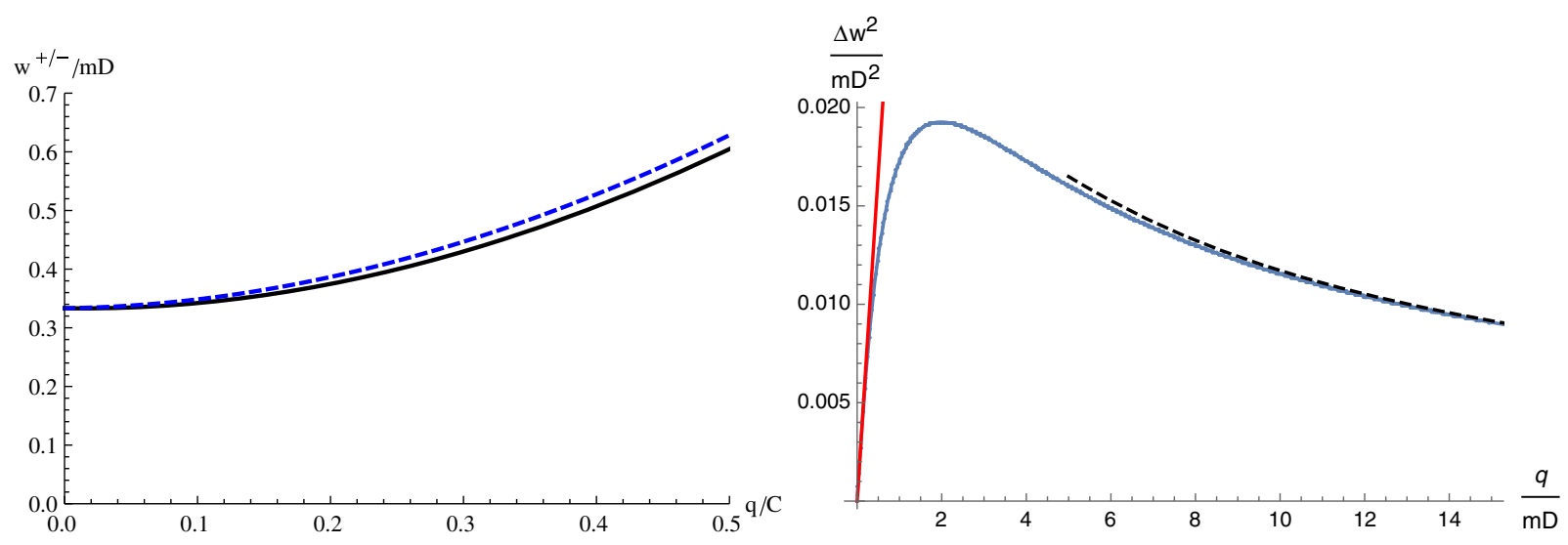

FIG. 1. (Left) Full numerical result for the real poles $\omega_{T, \pm}$ associated with the different photon polarizations as function of $q$ in units of $C$, showing the increase of the splitting between the two modes. The black solid line denotes the result for the positive helicity, while the blue dotted line refers to the negative helicity. (Right) Difference $\Delta \omega^{2}=\omega_{T,+}^{2}-\omega_{T,-}^{2}$ as function of the momentum $q$ in units of $m_{D}$. The red line denotes the analytical result for the difference computed for small $q$ from Eq. (15), while the black dashed one denotes the analytical result from large $q$ from Eq. (16). All these results are plotted for a fixed value $C=0.15 m_{D}$. 
for spacelike momenta, so that it cannot lead to processes with spontaneous radiation. Let us stress that the lowenergy theory used in Ref. [24] to describe chiral matter is only strictly valid in the static limit (see also [22,23]).

\section{FERMION DAMPING RATE}

We now consider a specific application of the perturbation theory developed in the previous section: the damping rate of a fermion in a chiral plasma. This can be obtained by explicitly computing the Feynman diagram associated with the scattering between the fermion and the plasma particles or, equivalently, from the imaginary part of the retarded fermion self-energy $\Sigma$ evaluated on shell [25]. As anticipated, in a chiral medium this quantity depends on its chirality $\chi$, and it can be expressed as

$\gamma_{\chi}(E)=-\left.\frac{1}{2 E} \operatorname{Tr}\left[\mathcal{P}_{\chi} \not \operatorname{Im} \Sigma\left(p_{0}+i \eta, \mathbf{p}\right)\right]\right|_{p^{0}=E}, \quad \eta \rightarrow 0^{+}$,

where $\mathcal{P}_{\chi}=\frac{1}{2}\left(1+\chi \gamma_{5}\right)$ are the chiral projectors, with the assignment $\chi= \pm 1$ for right- and left-handed fermions, respectively. The computation of the fermion self-energy
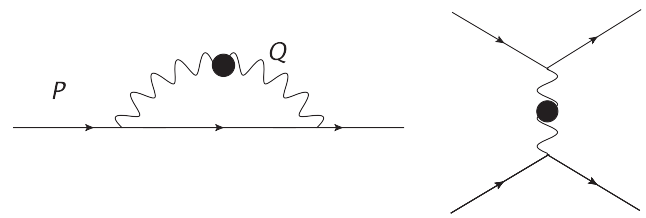

FIG. 2. (Left) Fermion self-energy entering the equation for the damping rate. (Right) Corresponding scattering process at $T=0$ : the energetic fermion interacts with particles from the medium via the exchange of Landau damped photons. The dot denotes a resummed propagator.

(Fig. 2, left) can be carried out, e.g., in the imaginary time formalism of thermal field theory and then analytically continued to Minkowski space. It is the same sort of computation as the one carried out in Refs. [13,14], although, as we will see, the presence of a chiral imbalance leads to an asymmetry between the left- and right-handed components of the fermion self-energy.

By following the same initial steps as in [13], while taking into account the new structure of the photon propagator in a chiral plasma, the damping rate can be expressed in terms of the fermion and photon spectral functions as

$$
\begin{aligned}
\gamma_{\chi}(E)= & -\frac{e^{2}}{2 E} \operatorname{Im} \int \frac{\mathrm{d}^{4} q}{(2 \pi)^{4}} \int_{-\infty}^{\infty} \frac{\mathrm{d} k_{0}}{2 \pi} \rho_{f}\left(k_{0}, k\right) \frac{1+f\left(q_{0}\right)-\tilde{f}\left(k_{0}-\mu_{\chi}\right)}{p_{0}-k_{0}-q_{0}+i \eta} \operatorname{Tr}\left(\mathcal{P}_{\chi} \not \gamma^{\mu} \not k \gamma^{\nu}\right) \\
& \times\left.\left[\delta_{\mu 0} \delta_{\nu 0} \rho_{L}\left(q_{0}, q\right)+\sum_{h= \pm} \mathcal{P}_{\mu \nu}^{T, h} \rho_{T}^{h}\left(q_{0}, q\right)\right]\right|_{p^{0}=E}
\end{aligned}
$$

where $k^{\mu}=\left(k^{0}, \mathbf{k}=\mathbf{p}-\mathbf{q}\right)$, where

$$
\rho_{f}\left(k_{0}, \mathbf{k}\right)=2 \pi \operatorname{sgn}\left(k_{0}\right) \delta\left(k_{0}^{2}-|\mathbf{k}|^{2}\right)
$$

is the fermion spectral function, and $f$ and $\tilde{f}$ are BoseEinstein and Fermi-Dirac distribution functions $(\beta=1 / T)$, respectively,

$f\left(q_{0}\right)=\frac{1}{\mathrm{e}^{\beta q_{0}}-1}, \quad \tilde{f}\left(k_{0}-\mu_{\chi}\right)=\frac{1}{\mathrm{e}^{\beta\left(k_{0}-\mu_{\chi}\right)}+1}$.

Equation (20) essentially gives an account of scattering (see Fig. 1, right) and/or annihilation processes of the external fermion with the particles of the medium, through the exchange of photons.
In the following, we will focus on the study of the ultradegenerate limit and set $T=0$, as in this case the imaginary part of the fermion self-energy is finite, after taking into account the resummation in the photon propagators. While at finite $T$ both fermion-fermion scattering and fermion-antifermion annihilation processes contribute to the damping, at $T=0$ only the first process is possible. Let us first concentrate on the value of the damping rate when the photon momentum is soft (that is, of the order of the Debye mass) and introduce an intermediate cutoff $q^{*}$, such that $m_{D} \ll q^{*} \ll m_{D} / e$. We also assume that $E \geq \mu_{L}, \mu_{R}$. Then, expanding $|\mathbf{k}|=E \sqrt{1-2 q \cos \theta / E+q^{2} / E^{2}}$ up to order $1 / E$, one reaches

$$
\begin{aligned}
\gamma_{\chi}^{\text {soft }} \simeq & \frac{e^{2}}{2} \int \frac{d^{3} q}{(2 \pi)^{3}}\left(\Theta\left(q_{0}\right)-\Theta\left(\mu_{\chi}-E+q_{0}\right)\right) \Theta\left(q^{*}-q\right) \\
& \times\left.\left\{\rho_{L}\left(q_{0}, q\right)\left(1-\frac{q_{0}}{E}\right)+\frac{1}{2}\left(1-\cos ^{2} \theta\right) \sum_{h= \pm}\left[\left(1-\frac{q^{0}}{E}-\chi h \frac{q}{E}\right) \rho_{T}^{h}\left(q_{0}, q\right)\right]\right\}\right|_{q_{0}=q \cos \theta},
\end{aligned}
$$


where we have kept terms up to order $1 / E$. For $\mu_{5}=0$, the leading term gives the same result obtained in Ref. [13] as then $\mu_{R}=\mu_{L}$, and also $\rho_{T}^{+}=\rho_{T}^{-}$. In the presence of chiral asymmetry, however, the leading term in the energy expansion still depends on the chirality of the fermion involved, both through the Pauli blocking effects encoded in $\mu_{\chi}$ and the $\mu_{5}$ dependence in the transverse photon spectral functions. At this point, we may already notice upon inspecting Eq. (23) that, at the Fermi surface, $E=\mu_{\chi}$, the damping rate vanishes. This is simply due to Pauli blocking, which is enforced by the occupation numbers, which at vanishing temperature reduce to sharp step functions. At zero temperature, the damping rate is thus found to be zero even in presence of a chiral imbalance. Of particular interest is the last term of Eq. (23), which shows that a fermion of definite chirality interacts differently with the transverse photons, depending on their polarization. While this effect is of order $1 / E$, it provides a qualitative new feature that may play an important role in chiral plasmas and deserves to be better explored. It is possible to derive the associated different $1 / E$ vertices of these couplings in a systematic way using the on-shell effective field theory proposed in [26].

We have checked that the expression given in Eq. (23) corresponds to the decay rate that might be computed by considering the scattering process of Fig. 2 (right), at leading order in the energy expansion, following a similar analysis as the one carried out in Ref. [27], but here at zero temperature.

In the imaginary part of the fermion self-energy, it is only $\rho^{<}$the Landau damping part of the spectral function contributes, as the kinematical constraints force the photon momentum to be spacelike $\left(q_{0}^{2} \leq q^{2}\right)$. After plugging into Eq. (23) the expressions derived in the previous section for the spectral functions, we arrive at

$$
\begin{aligned}
\gamma_{\chi}^{\text {soft }}(E)= & \frac{e^{2}}{8 \pi} \int_{D_{\chi}} d q_{0} d q\left\{\frac{q_{0} m_{D}^{2}}{\left[q^{2}+m_{D}^{2} Q_{1}(x)\right]^{2}+\frac{\pi^{2} m_{D}^{4} x^{2}}{4}}\left(1-\frac{q_{0}}{E}\right)\right. \\
& \left.+\sum_{h= \pm}\left[\frac{q^{0}\left(m_{D}^{2}+h C q\right)}{\frac{\pi^{2} x^{2}}{4}\left(h C q+m_{D}^{2}\right)^{2}+\left[2 q^{2}-\left(h C q+m_{D}^{2}\right) Q_{1}(x)+\frac{m_{D}^{2}}{1-x^{2}}\right]^{2}}\left(1-\frac{q^{0}}{E}-\chi h \frac{q}{E}\right)\right]\right\}+\mathcal{O}\left(\frac{1}{E^{2}}\right),
\end{aligned}
$$

where the domain of integration is

$$
D_{\chi}=\left\{0 \leq q_{0} \leq E-\mu_{\chi} ; q_{0} \leq q \leq q *\right\} .
$$

The hard contribution to the damping rate can be estimated from Eq. (23) using free or unresummed propagators (that is, setting $m_{D}=C=0$ in the spectral functions) and taking into account that the momentum transfer should obey $q>q *$, and $q<q_{\max }=2 E$, where $q_{\max }$ is the maximum momentum transfer allowed by the kinematics.

Let us remark here that by working at zero temperature we do not have the characteristic Bose enhancement at vanishing frequencies, and the presence of unstable modes does not lead to problems of nonintegrability or ill-behaved terms in the above integrals. This is similar to what occurs in the computation of energy loss in systems with Weibel instabilities, where one witnesses a sort of effective "dynamical shielding" of the unstable modes [28].

When extracting the leading contribution close to the Fermi surface, we find the same leading behavior as in chiral symmetric matter $[13,15,29]$, namely,

$$
\gamma_{\chi}(E)=\frac{e^{2}}{24 \pi}\left(E-\mu_{\chi}\right)+\mathcal{O}\left(\left(E-\mu_{\chi}\right)^{5 / 3}\right) .
$$

As a by-product, we can say that non-Fermi liquid behavior is then expected for the two chiral fermion populations. In Fig. 3, we show the result of the numerical integration of

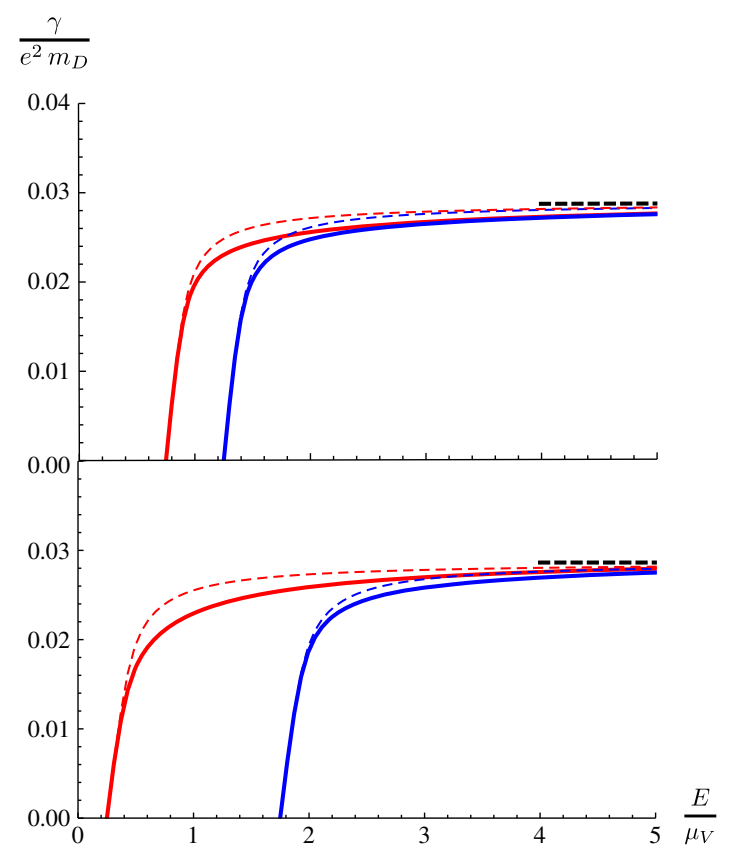

FIG. 3. Damping curves for left- (red lines) and right-handed (blue lines) fermions for a fixed ratio $\mu_{5} / \mu_{V}=1 / 4$ (top) and $3 / 4$ (bottom). The solid lines denote the result of the full numerical integration of Eq. (23) up to order $1 / E$, while the dashed lines show (for comparison only) the leading-order results without $1 / E$ corrections. The curves for both chiralities approach the limit $\gamma \sim 0.027 e^{2} m_{D}$, which is denoted as a black dashed line. 
Eq. (23) for left- and right-handed fermions for two fixed values of the ratio $\mu_{5} / \mu_{V}$ (we choose $\mu_{5}>0$ so that the density of right-handed fermions is larger than the lefthanded ones). The damping is zero at their respective Fermi surfaces $\left(E=\mu_{R, L}\right)$ and then grows with the energy and approaches the same limit for both chiralities. For a fixed value of the energy, the value of the damping for lefthanded fermions is always larger, indicating that for $\mu_{5}>0$ right-handed fermions have a longer lifetime compared to the left-handed ones. The inclusion of $1 / E$ effects provides a visible effect as we move away from the Fermi surface; more specifically, for a positive $\mu_{5}$ the damping rate for a left-handed fermion increases more slowly with energy than its right-handed counterpart. While being subleading, this effect becomes stronger as $\mu_{5}$ increases, as can be seen from the figure.

Let us comment at this point on the regime of validity of our result. The fact that the damping rate vanishes at the Fermi surface has been concluded from a perturbative computation. This result can be understood in terms of phase-space restrictions and Pauli blocking, as the imaginary part of the fermion self-energy can be written as a decay rate [25]. It is then possible to see that there is no phase space available for the scattering of a fermion at the Fermi surface with one in the Fermi sea producing two final states above the Fermi sea. As the inverse of the damping rate gives the lifetime of the quasiparticle, one could apparently conclude the stability of the Fermi sea. However, when the unstable gauge modes grow large, at timescales $t_{\text {ins }} \sim 1 /\left(\alpha^{2} \mu_{5}\right)$ [19], perturbation theory ceases to be valid. Thus, we can only conclude that the Fermi sea is stable at shorter timescales.

\section{CONCLUSIONS}

In this work, we have shown how to extend the HTL resummation scheme to a plasma in the presence of a chiral imbalance and discussed how the dispersion relations for the collective modes are altered by the presence of a chiral chemical potential. Aside from the appearance of imaginary poles, which may give rise to plasma instabilities, we investigated in detail the splitting of the collective modes associated with the different circular polarizations of the photons.

We then computed the damping rate of a fermion in a chiral plasma by taking into account effects of the chiral imbalance both in the fermion and the resummed photon propagators. The value of the damping rate was found to depend on the chirality of the fermion, unless its energy is much larger than the chemical potentials of the system, when a unique asymptotic value is reached. It is important to recall that our results should be valid for timescales shorter than the onset of the instability, as for larger times a perturbative analysis such as the one we have carried out ceases to be valid. For larger timescales, one should then derive an effective field theory to describe the system at those timescales in order to see how the chiral plasma instability affects the Fermi seas, similar to the approaches carried out to see the evolution of Weibel instabilities for non-Abelian plasmas in Ref. [30]. At finite temperature and at timescales where the hydrodynamical regime is reached, one can actually study the dynamical evolution of the chiral chemical potential by studying Maxwell's equations combined with the chiral anomaly equation $[31,32]$.

It would also be interesting to compute the fermion lifetime for nonvanishing values of the temperature. As for the chiral symmetric case, the Bose enhancement associated with the photon propagator in the fermion self-energy yields an infrared divergence, and further resummations of Feynman diagrams would then be required [12]. Furthermore, such an additional infrared enhancement together with the additional structure generated by the chiral imbalance might introduce additional issues of nonintegrability, and a careful investigation on whether the dynamical shielding still holds would be required.

Finally, while we have seen that already for a QED plasma the presence of a chiral imbalance induces significant new effects, it would also be interesting to explore the case of non-Abelian (QCD or electroweak) plasmas, especially for their cosmological implications. In the non-Abelian case, in order to preserve gauge invariance, the resummation techniques not only modify the gauge propagators but also the vertex functions whenever the external lines have soft momenta. The breaking of the $P$ and $C P$ symmetries suggests that the vertices would be different for the different gauge transverse polarized states, leading to different imprints of the chiral fermion imbalance.

\section{ACKNOWLEDGMENTS}

We thank J. Soto and J. Torres-Rincon for useful discussions. We have been supported by the MINECO (Spain) under the Projects No. FPA2016-81114-P and No. FPA2016-76005-C2-1-P, as well by the Generalitat de Catalunya by Project No. 2017-SGR-929 (Catalonia). This work was also supported by the COST Action CA15213 THOR. 
[1] D. E. Kharzeev, Prog. Part. Nucl. Phys. 75, 133 (2014).

[2] D. E. Kharzeev, J. Liao, S. A. Voloshin, and G. Wang, Prog. Part. Nucl. Phys. 88, 1 (2016).

[3] X. G. Huang, Rep. Prog. Phys. 79, 076302 (2016).

[4] K. Landsteiner, Acta Phys. Pol. B 47, 2617 (2016).

[5] E. V. Gorbar, V. A. Miransky, I. A. Shovkovy, and P. O. Sukhachov, Fiz. Nizk. Temp. 44, 635 (2018) [Low Temp. Phys. 44, 487 (2018)].

[6] M. Joyce and M. E. Shaposhnikov, Phys. Rev. Lett. 79, 1193 (1997).

[7] H. Tashiro, T. Vachaspati, and A. Vilenkin, Phys. Rev. D 86, 105033 (2012).

[8] A. Vilenkin, Phys. Rev. D 22, 3080 (1980).

[9] A. Vilenkin, Phys. Rev. D 20, 1807 (1979).

[10] K. Fukushima, D. E. Kharzeev, and H. J. Warringa, Phys. Rev. D 78, 074033 (2008).

[11] D. T. Son and P. Surowka, Phys. Rev. Lett. 103, 191601 (2009).

[12] J. P. Blaizot and E. Iancu, Phys. Rev. Lett. 76, 3080 (1996).

[13] M. Le Bellac and C. Manuel, Phys. Rev. D 55, 3215 (1997).

[14] C. Manuel, Phys. Rev. D 62, 076009 (2000).

[15] B. Vanderheyden and J. Y. Ollitrault, Phys. Rev. D 56, 5108 (1997).

[16] T. Holstein, R. Norton, and P. Pincus, Phys. Rev. B 8, 2649 (1973); M. Yu Reizer, Phys. Rev. B 40, 11571 (1989).

[17] E. Braaten and R. D. Pisarski, Nucl. Phys. B337, 569 (1990).

[18] M. L. Bellac, Thermal Field Theory (Cambridge University Press, Cambridge, England, 1996).
[19] Y. Akamatsu and N. Yamamoto, Phys. Rev. Lett. 111, 052002 (2013).

[20] S. Mrowczynski, B. Schenke, and M. Strickland, Phys. Rep. 682, 1 (2017).

[21] J. F. Nieves and P. B. Pal, Phys. Rev. D 39, 652 (1989); 40, 2148(E) (1989).

[22] M. Laine, J. High Energy Phys. 10 (2005) 056.

[23] C. Manuel and J. M. Torres-Rincon, Phys. Rev. D 89, 096002 (2014).

[24] K. Tuchin, Phys. Lett. B 786, 249 (2018).

[25] H. A. Weldon, Phys. Rev. D 28, 2007 (1983).

[26] C. Manuel and J. M. Torres-Rincon, Phys. Rev. D 90, 076007 (2014).

[27] J. P. Blaizot and E. Iancu, Phys. Rev. D 55, 973 (1997).

[28] P. Romatschke and M. Strickland, Phys. Rev. D 69, 065005 (2004).

[29] A. Gerhold and A. Rebhan, Phys. Rev. D 71, 085010 (2005).

[30] A. Rebhan, P. Romatschke, and M. Strickland, Phys. Rev. Lett. 94, 102303 (2005).

[31] A. Boyarsky, J. Frohlich, and O. Ruchayskiy, Phys. Rev. Lett. 108, 031301 (2012).

[32] C. Manuel and J. M. Torres-Rincon, Phys. Rev. D 92, 074018 (2015).

Correction: Equations (23) and (24) contained errors and have been fixed. 